The Israeli Journal of Aquaculture - Bamidgeh, IJA.73.2021.1401945, 15 pages

CCBY-NC-ND-4.0 • https://doi.org/10.46989/001c.24282

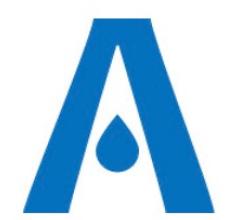

The $I J A$ is a peer-reviewed open-access, electronic journal, freely available without charge to users

Produced by the AquacultureHub non-profit Foundation Sale of $I J A$ papers is strictly forbidden

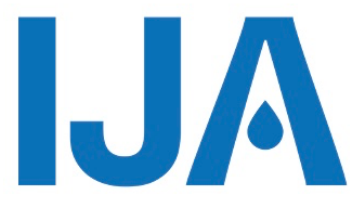

\title{
Effects of dietary starch levels on growth, feed utilization, glucose and lipid metabolism in non-transgenic and transgenic juvenile common carp (Cyprinus carpio L.)
}

\author{
Wei Guo ${ }^{1,2}$, Lele Fu ${ }^{1,2}$, Yunya $\mathrm{Wu}^{1,2}$, Haokun Liu ${ }^{1,3}$, Yunxia Yang ${ }^{1,3}$, Wei Hu${ }^{1,3}$, \\ Shouqi Xie ${ }^{1,2,3 *}$
}

\author{
${ }^{1}$ State Key Laboratory of Freshwater Ecology and Biotechnology, Institute of \\ Hydrobiology, Chinese Academy of Sciences, Wuhan, Hubei, 430072, P.R. China \\ 2 University of Chinese Academy of Sciences, Beijing, 100049 P.R. China \\ ${ }^{3}$ Hubei Engineering Research Center for Aquatic Animal Nutrition and Feed, Wuhan, \\ Hubei, PR China
}

Keywords: GH transgenic common carp, dietary starch levels, growth, feed utilization, gene expression

\begin{abstract}
This experiment investigated the effects of dietary starch on growth performance, feed utilization, glucose and lipid metabolism of non-transgenic and growth hormone $(\mathrm{GH})$ transgenic juvenile common carp (Cyprinus carpio L.). Transgenic common carp (TG fish) and non-transgenic (NT fish) were fed diets with five starch levels (0,10\%, 20\%, 30\%, 40\%). After 41 days, TG fish showed higher growth and feed utilization at low-starch diets, while similar growth, lower feeding rate (FR) and slightly higher feed efficiency (FE) was observed at high-starch diets. Lower plasma glucose level and hepatic glycogen content were observed in TG fish, which could be related to the higher glycolysis (high gene expression of hexokinase (hk), p < 0.01) and gluconeogenesis (high gene expression of fructose-1,6-bisphosphatase (fbpase), $p<0.01$; glucose6 -phosphatase (g6pase), p < 0.01) of TG fish. Compared to NT fish, weakened fat synthesis (lower gene expression of fatty-acid synthase (fas), $p<0.01$; acetyl-CoA carboxylase 1 (acc1), $\mathrm{p}<0.01$; acyl-CoA desaturase $1(\operatorname{scd} 1), \mathrm{p}=$ 0.011 ) and enhanced fatty acid oxidation (high gene expression of hormonesensitive lipase (hsl), p =0.01; carnitine palmitoyl transferase 1a (cpt-1a), p < 0.01 ), led to the decrease of body lipid content in TG fish. On the other hand, with increased dietary starch, increased body lipid and hepatic glycogen contents were observed in both TG and NT fish, suggesting that high dietary starch levels promoted glycolysis, fat synthesis and inhibited gluconeogenesis, fatty acid $\beta$-oxidation. Overall, TG fish showed higher growth performance at low starch diets, and higher ability of glycolysis, fatty acid oxidation and lower lipid synthesis than the NT fish.
\end{abstract}




\section{Introduction}

Dietary carbohydrates play different nutritional roles in different fishes (Wilson, 1994). But fish have limited ability to utilize dietary carbohydrates (Kamalam et al., 2017; Polakof et al., 2012). However, when carbohydrates are not enough in the diet, other nutrients such as protein and lipids are catabolized for energy to provide metabolic intermediates for the synthesis of other biologically important compounds (Wilson, 1994). Therefore, it is important to provide the appropriate carbohydrate in the diet for cultured fish. In a certain range, increased contents of carbohydrate in fish feed may show protein sparing effects (Mohanta et al., 2007). Some studies have shown that optimal carbohydrate diet can also promote the growth of fish at definite dietary protein and lipid (Hemre et al., 1998; Hung et al., 1989).

Growth hormone $(\mathrm{GH})$ gene transgenesis has been confirmed in several fish species to enhance growth (Cook et al., 2000; Fu et al., 2007; Nam et al., 2001; Rahman et al., 2001). GH transgenic common carp showed elevated growth by increased appetite and feed conversion efficiency (Zhong et al., 2012). Some studies have shown that GH transgenesis influenced metabolic reactions in coho salmon (Oncorhynchus kisutch) by improving carbohydrate degradation for energy production and lipid synthesis, and increasing utilization of lipids and proteins for body growth (Leggatt et al., 2009). Similarly, it has also been suggested that transgenic fish had somewhat modified regulation of glucose metabolism (Panserat et al., 2014). Therefore, it is necessary to investigate the carbohydrate utilization ability of transgenic and non-transgenic common carp.

The regulation of glucose metabolism is mainly achieved by glycolysis, gluconeogenesis, glycolipid transformation, glycogen synthesis, and degradation. The regulation of the glycolysis pathway is mainly dependent on key enzymes such as hexokinase (HK), glucokinase (GK), phosphofructokinase (PFK), and pyruvate kink (PK). In contrast, the regulation of gluconeogenesis is mainly depended on several key enzymes, such as phosphoenolpyruvate carboxykinase (PEPCK), fructose-1,6-bisphosphatase (FBPase) and glucose-6-phosphatase (G6Pase) (Saltiel, Kahn, 2001). It is also a vital glucose homeostasis pathway by increasing fat synthesis and glycogen synthesis activity after high dietary carbohydrate intake in fish (Suárez et al., 2002). This study was designed to evaluate the differences in carbohydrate metabolism between transgenic and nontransgenic common carp and find the effects of $\mathrm{GH}$ transgenesis on fish glucose metabolism.

\section{Materials and Methods}

Experimental diets, experimental fish, and feeding trial

The formulation and chemical composition of experimental diets are shown in Table 1. Fish meal, soybean meal and casein were used as major dietary proteins, and fish oil and soybean oil as lipid sources. Five experimental diets were formulated by supplementing of increasing corn starch as a carbohydrate to obtain graded starch levels $(0,10 \%, 20 \%$, $30 \%, 40 \%$ ). All ingredients passed through a $375 \mu \mathrm{m}$ sieve before entirely mixed. The wet dough was extruded into $3.6 \mathrm{~mm}$ pellets by a laboratory extruder (SLP-45, Fishery Mechanical Facility Research Institute, Shanghai, China). The pellets were oven-dried at $70{ }^{\circ} \mathrm{C}$ and stored at $-20^{\circ} \mathrm{C}$ until used.

The experimental fish including TG fish and NT fish of same days after hatching were obtained from the Huanghu Lake Experimental Station of the Institute of Hydrobiology, Chinese Academy of Sciences (Susong, Anhui, China). Before the trial, all fish fed for 2 weeks to acclimate the experimental condition. At the beginning of the trial, all fish were fasted for $24 \mathrm{~h}$. Apparent healthy TG fish (initial weight $30.5 \pm 0.06 \mathrm{~g}$ ) and NT fish (initial weight $17.1 \pm 0.08 \mathrm{~g}$ ) with similar size were selected, then batch weighted and distributed into round fiberglass tanks (diameter: $70 \mathrm{~cm}$; water volume: $400 \mathrm{~L}$ ) at a density of 15 fish per tank. Each experimental diet-fish strain was randomly assigned to triplicate tanks. Fish were fed to apparent satiation twice a day (8:30 and 16:30). The feces were removed by siphoning before each feeding. 
During the experiment, each tank received continuous aeration. Water temperature was recorded daily and was $30.85 \pm 0.31^{\circ} \mathrm{C}$. Light intensity was about $2.9 \mu \mathrm{mol} \mathrm{m}^{-2} \mathrm{~s}^{-1}$ at the water surface, and the light period was from 8:00 to 20:00. The water flowing rate into each tank was approximately $5 \mathrm{~L} \mathrm{~min}-1$. Dissolved oxygen was above $6.0 \mathrm{mg} \mathrm{L}^{-1}$ and $\mathrm{pH}$ was 6.7-7.0. Ammonia nitrogen was less than $0.4 \mathrm{mg} \mathrm{L}^{-1}$.

\section{Sample collection}

At the start of the trial, triplicate groups of three fish were sampled randomly for initial body composition analysis. After 41 days of the feeding trial, fish were anesthetized by MS-222 solution ( $100 \mathrm{mg} \mathrm{L}-1$ ) after $24 \mathrm{~h}$ fasting, then were bulk weighted to evaluate the feeding rate (FR), specific growth rate (SGR), and feed efficiency (FE). Two fish of each tank were sampled and frozen at $-20^{\circ} \mathrm{C}$ for the determination of whole-body composition. Two blood samples were collected using heparinized syringes from the caudal vein of two fish in each tank, and the blood samples were centrifuged $\left(3500 \mathrm{~g}, 10 \mathrm{~min}, 4^{\circ} \mathrm{C}\right.$; Eppendorf 5417R; Vertrieb, Germany) to obtain plasma samples. After blood sampling, the liver and muscle were dissected on ice, and then samples were stored at $-80^{\circ} \mathrm{C}$ until analyzed.

\section{Chemical analysis}

Proximate composition analysis of all samples was conducted using the methods described by AOAC (2003). Moisture content was determined by oven drying at $105^{\circ} \mathrm{C}$ to constant weight. Crude protein content was determined by Kjeldahl method after acid digestion using 2300 Kjeltec Analyzer Unit (FOSS Tecator, Haganas, Sweden). Crude lipid content was determined by chloroform-methanol extraction. Ash was determined by combustion in muffle furnace at $550^{\circ} \mathrm{C}$.

Plasma alanine aminotransferase (ALT), aspartate aminotransferase (AST), triglyceride, total cholesterol (TC), glucose (Glu), HDL-Cholesterol (HDL-C), LDLCholesterol (LDL-C) were tested by automatic biochemistry analyzer BS-460 produced by Mindray, using standard kits according to the instructions (Mindary Bio Medical Electronic Limited by Share Ltd, Shenzhen, China). Tissue glycogen (liver and muscle) was determined using the amyloglucosidase method, and the reagents were prepared by Nanjing Jiancheng Bioengineering Institute, Nanjing, China.

\section{Gene expression analysis}

Total RNAs were extracted from the liver tissue using TRIzol reagent (Invitrogen, Carlsbad, California, USA), and CDNA was reverse transcribed using an M-MLV First-Strand Synthesis Kit (Invitrogen, Shanghai, China), following the manufacturer's instructions. Quantitative reverse transcription-polymerase chain reaction (RT-PCR) was performed on a LightCycler 480 II (Roche Diagnostics, Basel, Switzerland), using SYBR Green I Master Mix (Roche Diagnostics, Indianapolis, IN, USA). Quantitative real-time RT-PCR determining target gene expression. The primers used in this study are listed in Table $\mathbf{2}$ were designed based on the obtained fragment cDNA sequences. $\beta$-actin was chosen as internal reference for normalization. Expression levels were calculated according to Vandesompele et al. (2002)

\section{Statistical analysis}

Data were analysed with SPSS 18.0 (SPSS, IL, USA) and were expressed as means \pm S.E. A two-way analysis of covariance (ANCOVA) was performed, with initial body weight as a concomitant variable. Where appropriate, differences between strains or diets were analyzed using one-way ANCOVAs. The probability level of statistical significance was $p<$ 0.05 . 
Table 1 Formulation and proximate composition of experimental diets (\% dry matter)

\begin{tabular}{|c|c|c|c|c|c|}
\hline \multirow{2}{*}{ Ingredients } & \multicolumn{5}{|c|}{ Dietary starch level (\%) } \\
\hline & 0 & 10 & 20 & 30 & 40 \\
\hline White fishmeala & 10 & 10 & 10 & 10 & 10 \\
\hline Soybean meal & 10 & 10 & 10 & 10 & 10 \\
\hline Casein ${ }^{b}$ & 21.5 & 21.5 & 21.5 & 21.5 & 21.5 \\
\hline Corn starch & 0 & 10 & 20 & 30 & 40 \\
\hline Fish oil & 3.3 & 3.3 & 3.3 & 3.3 & 3.3 \\
\hline Soybean oil & 3.3 & 3.3 & 3.3 & 3.3 & 3.3 \\
\hline $\begin{array}{l}\text { Carboxymethyl } \\
\text { cellulose sodium }\end{array}$ & 3 & 3 & 3 & 3 & 3 \\
\hline Cellulose & 43.38 & 33.38 & 23.38 & 13.38 & 3.38 \\
\hline Choline chloride & 0.11 & 0.11 & 0.11 & 0.11 & 0.11 \\
\hline Vitamin premix ${ }^{c}$ & 0.39 & 0.39 & 0.39 & 0.39 & 0.39 \\
\hline Mineral premix ${ }^{d}$ & 5 & 5 & 5 & 5 & 5 \\
\hline Ethoxyquin & 0.02 & 0.02 & 0.02 & 0.02 & 0.02 \\
\hline \multicolumn{6}{|c|}{ Proximate composition (\% dry matter) } \\
\hline Moisture & 9.85 & 10.01 & 10.92 & 11.65 & 11.06 \\
\hline Crude protein & 34.23 & 33.79 & 33.77 & 33.92 & 34.03 \\
\hline Crude lipid & 7.92 & 8.05 & 8.13 & 7.86 & 8.14 \\
\hline Ash & 7.35 & 7.27 & 7.24 & 7.19 & 7.08 \\
\hline $\begin{array}{l}\text { Gross energy (kJ } \\
\mathrm{g}-1 \text { dry matter) }\end{array}$ & 11.28 & 12.98 & 14.69 & 16.37 & 18.11 \\
\hline
\end{tabular}

a Pollock fishmeal from American Seafood Company, Seattle, Washington, USA.

b Purchased from Lanzhou Longruan Casein Co., Ltd., Lanzhou, Gansu, China.

c Vitamin premix (mg kg-1 diet): Thiamin, 20; Riboflavin, 20; Pyridoxine, 20; Cyanocobalamine, 0.02; Folic acid, 5; Calcium pantothenate, 50; Inositol, 100; Niacin, 100; Biotin, 0.1; Cellulose, 3412; Ascorbic acid, 100; Vitamin A, 11; Vitamin D, 2; Vitamin E, 50; Vitamin K, 10.

d Mineral premix (mg kg-1 diet): $\mathrm{NaCl}, 500 ; \mathrm{MgSO}_{4} \cdot 7 \mathrm{H}_{2} \mathrm{O}, 8155.6 ; \mathrm{NaH}_{2} \mathrm{PO}_{4} \cdot 2 \mathrm{H}_{2} \mathrm{O}, 12,500.0 ; \mathrm{KH}_{2} \mathrm{PO}_{4}$, 16,000.0; $\mathrm{CaHPO}_{4} \cdot 2 \mathrm{H}_{2} \mathrm{O}$ ，7650.6; $\mathrm{FeSO}_{4} \cdot 7 \mathrm{H}_{2} \mathrm{O}, 2286.2 ; \mathrm{C}_{6} \mathrm{H}_{10} \mathrm{CaO}_{6} \cdot 5 \mathrm{H}_{2} \mathrm{O}, 1750.0 ; \mathrm{ZnSO}_{4} \cdot 7 \mathrm{H}_{2} \mathrm{O}$, 178.0; $\mathrm{MnSO}_{4} \cdot \mathrm{H}_{2} \mathrm{O}, 61.4 ; \mathrm{CuSO}_{4} \cdot 5 \mathrm{H}_{2} \mathrm{O}, 15.5 ; \mathrm{CoSO}_{4} \cdot 7 \mathrm{H}_{2} \mathrm{O}, 0.91 ; \mathrm{KI}, 1.5$.

Table 2 Primer sequences used in this study

\begin{tabular}{llll}
\hline $\begin{array}{l}\text { Gene } \\
\text { name }\end{array}$ & Accession no. & $\mathrm{F}\left(5^{\prime}-3^{\prime}\right)$ & $\mathrm{R}\left(5^{\prime}-3^{\prime}\right)$ \\
\hline pfk & XM019107418.1 & CACGTACAAGCTGTTAGCT & TCGAAGCCATCATGGACGGT \\
hk & XM019097063.1 & CTTGTGAGACTCATCCTGCT & CAGACATGCTGGACAGCAAT \\
fbpase & XM019099298.1 & ACAGTCTGAATGAAGGCTAC & CTCATACAACAGCCTCAGCT \\
g6pase & XM019101462.1 & GCAGGTCAATCTCACTGGCT & CTGATGTAGTGGAGCGCTAT \\
acc1 & XM021476200.1 & GAGGAATCTGTGCGCTCCAT & ATGCCTGGAACATGATCTGT \\
fas & KY378913.1 & TAGAGAACCTGACTTCAGCT & TAGGACTTCTCAGCATGAC \\
scd1 & U31864.2 & TTCGTCACCTTCAGCGCTAT & CGCTTCTCTGGACACACGCT \\
hsl & XM_019124843.1 & AATGTCTCACGGTGTACGTG & ACTCGTACCAGCATAGGCAT \\
Ipl & KJ141167.1 & GTTACAGGCTGAGATTGACT & AGAACCGCGAGTGTTGGTGT \\
cpt-1a & XM019122007.1 & CTATCTGGAGTGACATCATG & CTCACTCACAGGTAGAGATG \\
aco3 & KR706500.1 & ACGGCCACTCATTCAGTGGT & CCGAGGTATCCTCACATTGT \\
$\beta-$-actin & JQ619774.1 & GATGATGAAATTGCCGCACTG & ACCAACCATGACACCCTGATGT \\
\hline
\end{tabular}


Growth and feed utilization

\section{Results}

Results of growth and feed utilization in two strains fed different starch levels are shown in Table 3. SGR, FE, and PER of TG fish were significantly higher than NT fish in low-starch diets $(P<0.05)$, and SGR of TG fish first increased and then decreased with increasing dietary starch levels. SGR of NT fish first increased and then tended to keep constant with increasing dietary starch levels $(P<0.05)$. FE and PER of both strains also first increased and then tended to keep constant with increasing dietary starch levels, FR showed no apparent differences between two strains, and FR of TG fish were not affected by dietary starch levels $(P>0.05)$, while FR of NT fish decreased with the increase starch levels $(P<$ 0.05). PRE also showed no obvious differences between the two strains $(P>0.05)$, but PRE was significantly lower in the no starch group than in other groups $(P<0.05)$.

\section{Body composition and tissue glycogen contents}

Table 4 showed the whole-body composition, and tissue glycogen contents of the fish fed different diets. Whole-body moisture of TG fish was significantly higher than NT fish ( $P$ $<0.05$ ), and whole-body moisture of both strains decreased significantly with the increased dietary starch levels $(P<0.05)$. Whole-body protein, lipid, and ash contents of TG fish were significantly lower than NT fish at different dietary starch levels. The difference in body lipid contents was apparent, especially in the low-starch diets $(P<0.05)$. Wholebody protein contents of TG fish were not affected by dietary starch levels $(P>0.05)$, but whole-body protein contents of NT fish decreased significantly with the increased dietary starch levels $(P<0.05)$. Whole-body ash contents of both strains decreased significantly with the increased dietary starch levels $(P<0.05)$, while whole body lipid contents of both strains increased with increasing dietary starch levels $(P<0.05)$. TG fish had significantly higher levels of liver glycogen than NT fish $(P<0.05)$, while no significant difference was observed in muscle glycogen $(P>0.05)$. However, with the increased dietary starch, tissue glycogen (liver and muscle) contents increased significantly $(P<0.05)$.

\section{Plasma physio-biochemical indices}

As shown in Table 5, ALT of TG fish were significantly lower than NT fish $(P<0.05)$, plasma AST of two strains had no significant difference in different dietary starch levels ( $P$ $>0.05)$, and not affected by dietary starch levels $(P>0.05)$. No significant difference was found between TG fish and NT fish in plasma triglycerides, TC, HDL-C and LDL-C (P > $0.05)$, while triglycerides contents of both strains first increased and then decreased with increasing dietary starch levels ( $P>0.05), T C, H D L-C$ and $L D L-C$ content of both strains increased with increasing dietary starch levels $(P<0.05)$. TG fish had significantly lower levels of plasma Glu than NT fish $(P<0.05)$, but no significant difference was found between diets $(P>0.05)$. 


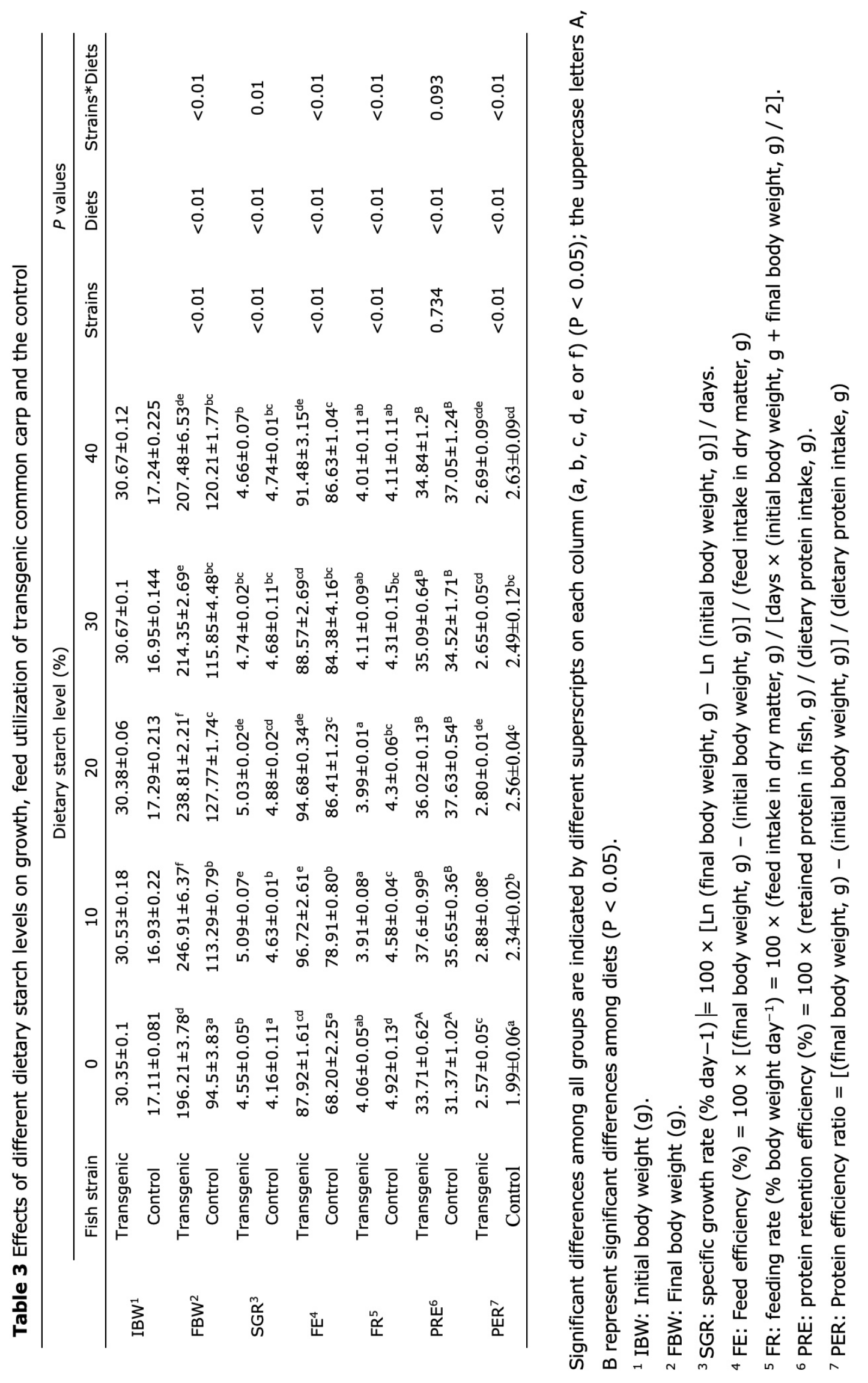




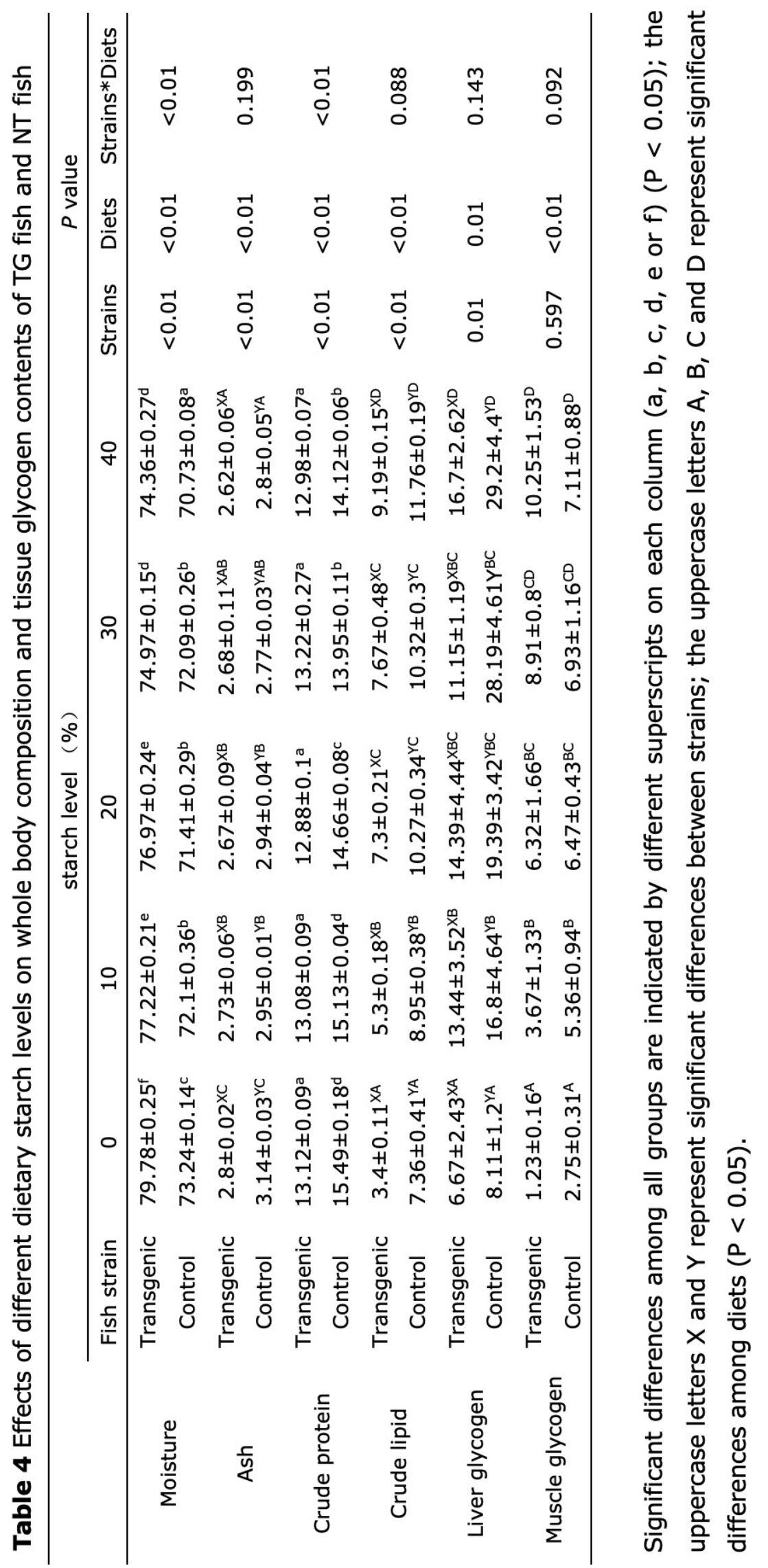




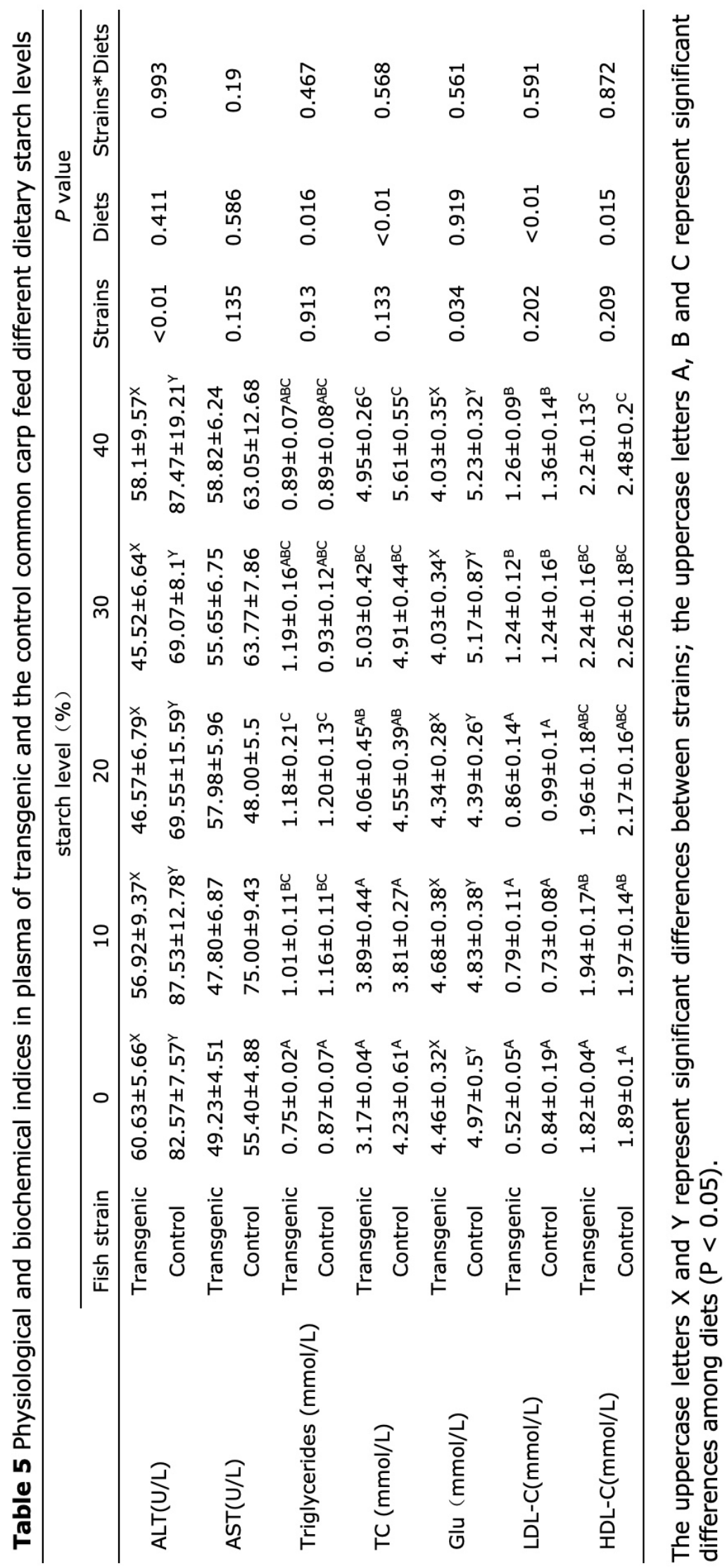




\section{Gene expression}

Figure 1 showed that the expression of liver genes related to glucose metabolism. The transcriptional levels of glycolysis-related gene hk was significantly up-regulated in TG fish $(P<0.05)$, pfk had no change $(P>0.05)$. The expression of hk and pfk of both strains significantly up-regulated with the increasing dietary starch levels $(P<0.05)$. Gluconeogenesis-related genes fbpase and g6pase significantly up-regulated in TG fish than in NT fish $(P<0.05)$. The expression of fbpase was significantly down-regulated with increasing dietary starch levels $(P<0.05)$. There were no differences in the expression of g6pase $(P>0.05)$. The transcriptional levels of the genes involved in the lipid synthesis pathway were determined, no differences in the expression of acc1 were detected in both strains $(P>0.05)$. However, fas and scd1 in TG fish displayed higher expressions than NT fish $(P>0.05)$. The expression of fas, acc1 and scd1 in both strains were significantly upregulated with the increasing dietary starch levels $(P<0.05)$. The expression of fatty acid oxidation-related genes includes hsl and cpt-1a significantly up-regulated in TG fish than in NT fish $(P<0.05)$, Ipl, and aco3 did not show significant differences between two strains $(P>0.05)$. The expression of Ipl, cpt-1a and aco3 in both strains were significantly downregulated with the increasing dietary starch levels $(P<0.05)$. The gene expressions of hsl were not affected by dietary starch levels $(P>0.05)$.
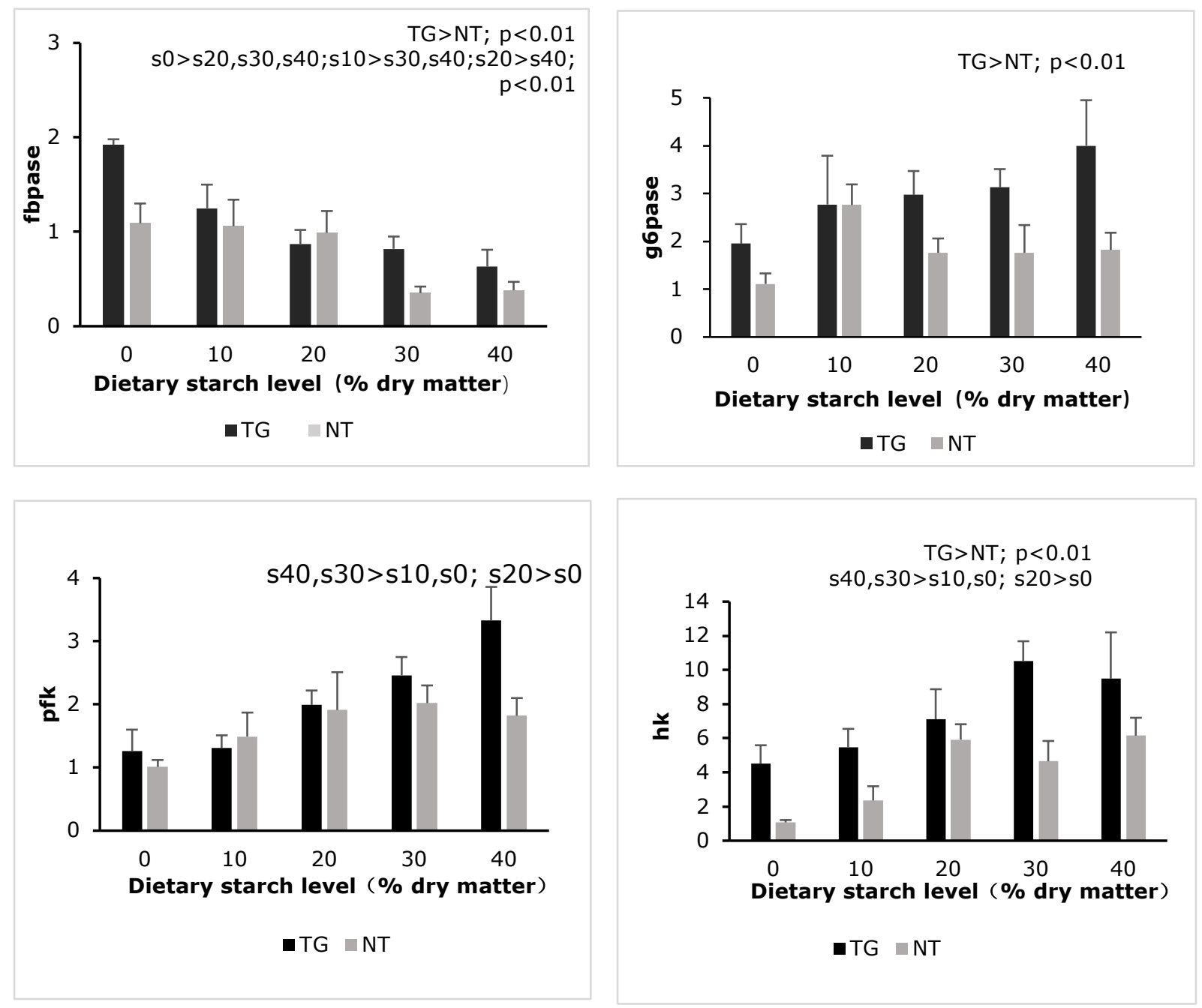

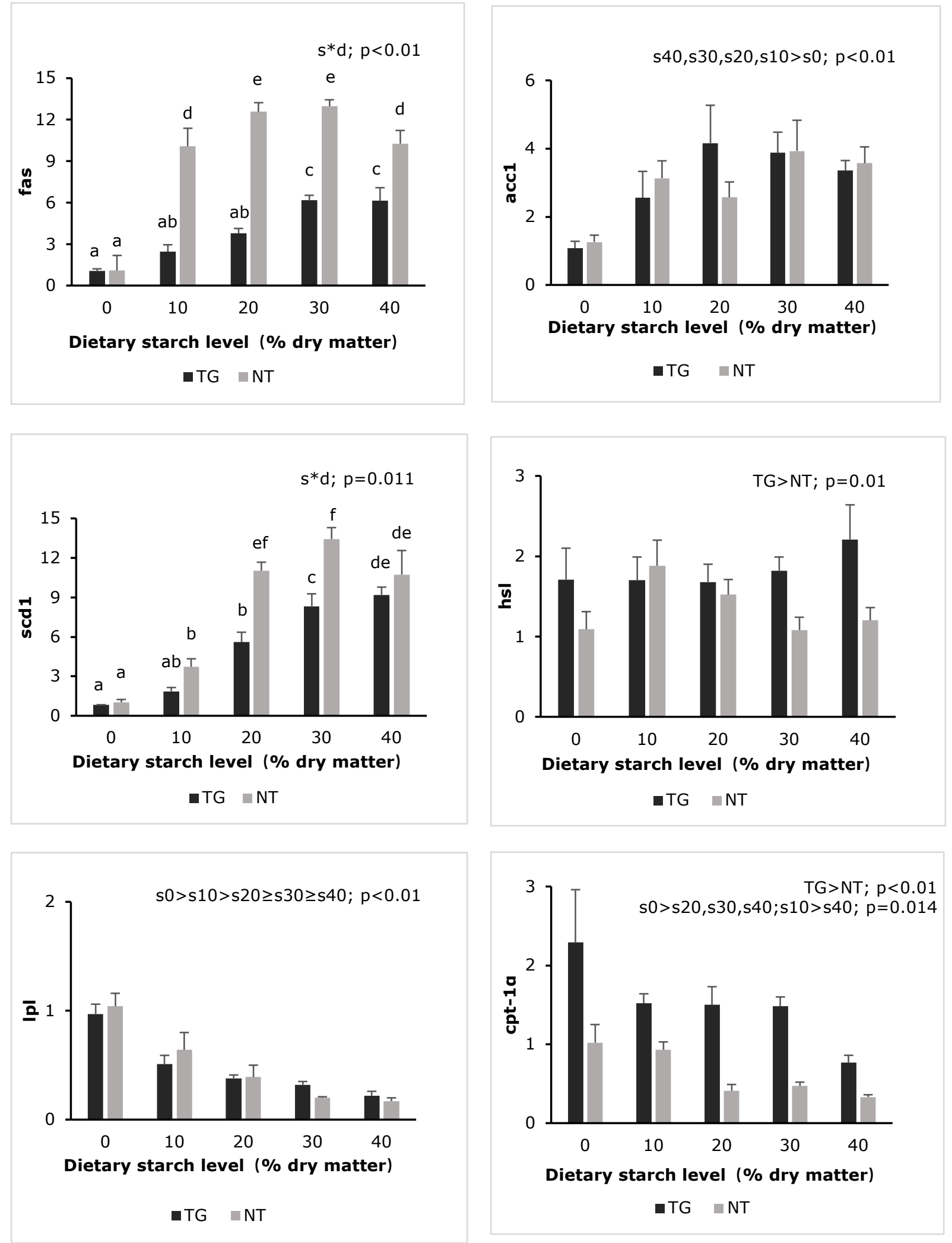


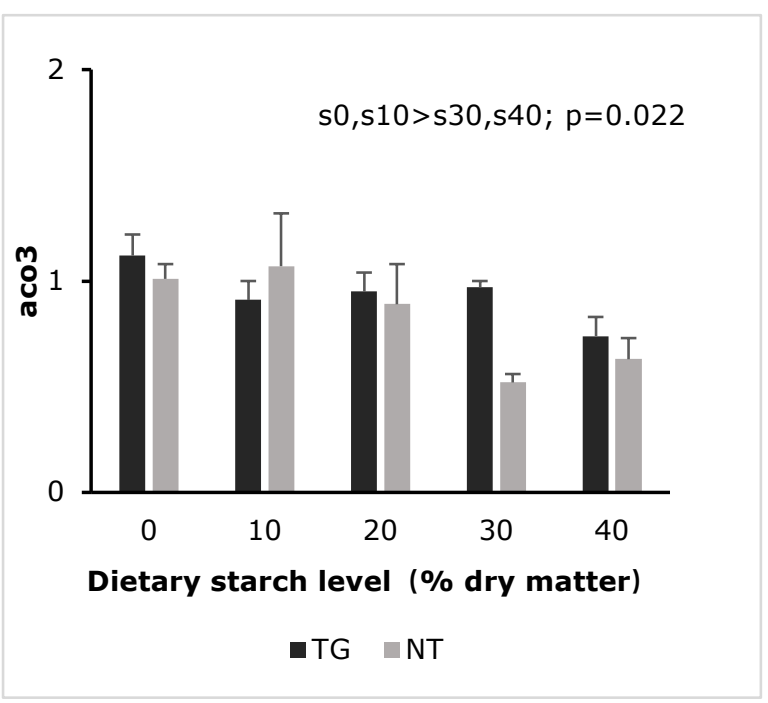

Figure 1 Relative expression of hepatic fbpase, g6pase pfk, hk, acc1, fas, scd1, Ipl, hsl,cpt-1a and aco3 genes of both strains fed diets with different starch levels (s0: 0\% starch, s10: $10 \%$ starch, s20: $20 \%$ starch, s30: 30\%starch, s40:40\% starch). Results represent the mean $\pm S E(n=6)$. Different superscripts $(a, b, c, d$, e or $f)$ within a column indicate significant $(P<0.05)$ difference between treatments.

\section{Discussion}

Common carp is a typical omnivorous fish, the present study found that TG fish had higher growth and feed efficiency than NT fish. The results were consistent with previous studies on transgenic fish, such as the transgenic common carp (Fu et al., 2007), transgenic salmonids (Salmo salar) (Cook et al., 2000), transgenic mud loaches (Misgurnus mizolepis) (Nam et al., 2001), transgenic Nile tilapia (Oreochromis niloticus) (Rahman et al., 2001). GH transgenic fish grow faster was the embodiment of a high concentration of growth hormone promoting growth. Carbohydrate is one of the dietary nutrients that profoundly affects feed utilization. The present study found that a certain amount of dietary starch increased feed efficiency and growth of TG fish. In previous studies, improved growth and feed utilization with increasing dietary starch levels were also reported in many other fish species (Li et al., 2016; Zhang et al., 2009). However, the optimal dietary starch level of TG fish is lower than that of NT fish. In the present study, TG fish's growth tended to decrease when the dietary starch level was high, which showed the effects on carbohydrate metabolism of GH transgenesis were different at high dietary starch. In the present study, the protein retention efficiency of TG fish increased first and then decreased with the increase in dietary starch level, which was consistent with the growth trend. The present study showed that TG fish had a higher protein efficiency ratio than NT fish, which reflected that TG fish could increase unit protein production compared with NT fish. Increased protein efficiency ratio has also been reported in transgenic coho salmon (Higgs et al., 2009). High starch treatment groups had significantly higher levels of protein efficiency ratio in both strains. Similar results were also found in mirror carp (Cyprinus carpio) (Li et al., 2015), hybrid grouper (male Epinephelus lanceolatus $\times$ female E. fuscoguttatus) (Li et al., 2019). It suggested that dietary carbohydrate supplied energy to obtain the proteinsparing effect. On the other hand, it was also reported that high starch diets resulted in decreased PER in largemouth bass (Micropterus salmoides) (Ma et al., 2019). It might be due to the fact that largemouth bass is a carnivorous fish, and high starch caused fatty liver and affected the health of the fish (Li et al., 2020; Ma et al., 2019).

Carbohydrate is an important energy source and excessive dietary carbohydrate usually resulted in fish crude lipid accumulation (Higgs et al., 2009). It is also confirmed that whole fish body lipid content increased with increasing dietary starch levels. The TG fish in the 
present study had lower body fat than NT fish. It could be due to the higher total carcass moisture content of TG fish and different fat metabolism in TG fish. Compared with NT fish, body protein in the wet weight of TG fish was significantly lower. The result was consistent with a previous study in the transgenic common carp (Fu et al., 2007). Higher total carcass moisture content, lower fat and protein content in transgenic Atlantic salmon were also observed (Cook et al, 2000). To adapt to a high carbohydrate diet, fish can store excess glucose in the form of glycogen. Excessive dietary carbohydrate was reported to increase fish glycogen content (Lee et al., 2004). The present study indicated that liver and muscle glycogen contents significantly increased with increasing dietary starch levels, and the same results were also observed in hybrid grouper (Epinephelus fuscoguttatus $q \times E$. lanceolatus $\hat{\delta}$ ) (Li et al., 2019), golden pompano (Zhou et al., 2015), Nile tilapia (Boonanuntanasarn et al., 2018), gibel carp (Carassius gibelio) (Song et al 2019). grass carp (Ctenopharyngodon idellus) and Chinese longsnout catfish (Leiocassis longirostris Günther) (Su et al., 2020). Interestingly, the liver glycogen content of TG fish was significantly lower than NT fish, while no significant difference was observed in muscle glycogen. The decrease of liver glycogen content may be due to the enhancement of glycolysis in TG fish.

In the present study, the plasma glucose levels in both strains were not affected by the diets. TG fish and NT fish showed sound control on plasma glucose levels at $40 \%$ dietary carbohydrate, and the same results were also observed in grass carp (Li et al., 2012), gible carp (Song et al., 2019). In this study, plasma TC, HDL-C and LDL-C concentration were positively correlated with dietary starch contents. Also, the concentration of triglyceride in plasma also increased first and then decreased slightly with increasing dietary starch levels, which suggested higher plasma lipids in response to high dietary starch levels. It is consistent with the high-fat content of fish caused by high starch diets. The de novo synthesis of lipid from carbohydrate was also found in other studies ( $\mathrm{Li}$ et al., 2015; Mohanta et al., 2009). After transporting into the cell, glucose could be used by glycolysis. In the present study, higher mRNA levels of $h k$ and $p f k$ encoding for the glycolytic enzymes were observed in the high starch diets. Glucose also could be produced by gluconeogenesis. The present study found that lower mRNA levels of fbpase were observed in the high starch diets, suggesting an efficient control on gluconeogenesis in response to high carbohydrate intake. It showed that TG fish and NT fish could control plasma glucose levels in both glycolysis and gluconeogenesis. Similar results were found in sea cucumber (Apostichopus japonicas) (Xia et al., 2015), golden pompano (Trachinotus ovatus) (Zhou et al., 2015). In the present study, higher mRNA levels of $h k$ fbpase and g6pase were observed in TG fish than NT fish. It indicates that the glycolysis potential and gluconeogenesis potential of TG fish were higher than that of NT fish. Higher glycolysis potential may be the main reason for the lower plasma glucose level of TG fish than NT fish. Panserat et al. (2014) reported GH transgenic coho salmon showed higher mRNA levels of G6PDH (glucose-6-phosphate dehydrogenase) and GK (glucokinase) in the liver, and GLUT4 (glucose transporter) in muscle, suggesting the potential for better use of glucose in $\mathrm{GH}$ transgenic coho salmon. Leggatt et al. (2009) reported transgenic fish had higher activities of glycolytic enzymes in white muscle. Transgenic fish was reported to prefer to get energy from carbohydrates (Higgs et al., 2009). Studies have shown that the fat accumulation in fish increased with increased dietary carbohydrate levels ( $\mathrm{Li}$ et al., 2012). The activity of the fatty synthase (FAS) in Atlantic salmon increased with the increase of dietary carbohydrate levels (Hemre et al., 1998). It was found in grass carp that fatty acid synthase (fas) and acetyl coenzymeA carboxylase (acc1) genes were significantly up-regulated, and lipoprotein lipase and carnitine palmitoyltransferase 1 (cpt1) gene expression were down-regulated in high dietary carbohydrate group (Cai et al., 2018). In the present study, the liver transcriptional levels of fas, acc1, scd1 significantly up-regulated and $I p I$, aco3, cpt-1a were significantly down-regulated with the increasing dietary starch levels in both strains. It was similar to the previous study and suggested that glucose homeostasis regulation by increasing lipid synthesis and reducing fatty acid oxidation was also a meaningful way to regulate glucose 
(Suárez et al., 2002). Zhang et al. (2013) found that growth hormone inhibited adipocyte synthesis and differentiation in mice. In the present study, TG fish had lower mRNA levels of fas, scd 1 than NT fish, and hsl, cpt-1a were higher expressed, this indicated that TG fish had lower fat synthesis potential and higher lipid oxidation potential compared with NT fish. It very well explained that the accumulation of crude lipid in TG fish was lower than NT fish. These results showed that growth hormone could inhibit fat synthesis and promote fatty acid oxidation. However, it has also been reported that the activity of lipolytic enzyme decreased and the activity of lipid synthase increased in transgenic coho salmon, indicating sparing of lipids (Higgs et al., 2009). It was not consistent with $\mathrm{GH}$ transgenic common carp results, indicating that the carbohydrate de novo lipid synthesis mechanism in GH transgenic common carp and GH transgenic coho salmon was different. The effect of $\mathrm{GH}$ on lipid accumulation was complex, warranting further investigation. In conclusion, the present study confirmed that TG fish and NT fish showed somecarbohydrate utilization differences. The lower level of whole-body lipid, liver glycogen, glycemic, lower fas, scd1 gene expressions, higher hk, fbpase, g6pase, and hsl cpt-1a suggested that TG fish had a somewhat modified regulation of glucose metabolism. TG fish may have more vital ability of glucose utilization (glycolysis and gluconeogenesis). Fat synthesis of TG fish decreased while the oxidation ability of fatty acid $\beta$ increased, which led to the decrease of lipid contents in TG fish. Extension studies should be focused on the effects of GH on glucose regulation at high dietary starch, especially in the transgenic carps after gonadal development.

\section{Acknowledgements}

This work was supported by the National Natural Science Foundation of China (31672661). The authors wish to thank Mr. Guanghan Nie for his technical assistance.

\section{References}

Boonanuntanasarn, S., Kumkhong, S., Yoohat, K., Plagnes-Juan, E., Burel, C., Marandel, L., Panserat, S.J.A., 2018. Molecular responses of Nile tilapia (Oreochromis niloticus) to different levels of dietary carbohydrates. Aquaculture, 482: 117-123. https://10.1016/j.aquaculture.2017.09.032

Cai, W.J., Liang, X.F., Yuan, X.C., Liu, L.W., 2018. Different strategies of grass carp (Ctenopharyngodon idella) responding to insufficient or excessive dietary carbohydrate. Aquaculture, 497: 292-298. https://10.1016/j.aquaculture.2018.07.042

Cook, J.T., Mcniven, M.A., Richardson, G.F., Sutterlin, A.M., 2000. Growth rate, body composition and feed digestibility/conversion of growth-enhanced transgenic Atlantic salmon (Salmo salar). Aquaculture, 188: 15-32. https://10.1016/S0044-8486(00)00331-84

Fu, C., Li, D., Hu, W., Wang, Y., Zhu, Z., 2007. Growth and energy budget of F-2 'all-fish' growth hormone gene transgenic common carp. Journal of Fish Biology, 70(2): 347-361. https://10.1111/j.1095-8649.2007.01301.x

Hemre, G.I., Hansen, T., 1998. Utilisation of different dietary starch sources and tolerance to glucose loading in Atlantic salmon (Salmo salar), during parr- smolt transformation. Aquaculture, 161(1): 145-157. https://10.1016/S0044-8486(97)00266-4

Higgs, D.A., Sutton, J.N., Kim, H., Oakes, J.D., Smith J., Biagi, C., Rowshandeli, M., Devlin, R.H., 2009. Influence of dietary concentrations of protein, lipid and carbohydrate on growth, protein and energy utilization, body composition, and plasma titres of growth hormone and insulin-like growth factor-1 in non-transgenic and growth hormone transgenic coho salmon. Aquaculture, 286(12): 27-137. https://10.1016/j.aquaculture.2008.08.036

Hung, S.S.O., Fynn-Aikins, F.K., Lutes, P.B., Xu, R., 1989. Ability of Juvenile White Sturgeon (Acipenser transmontanus) to Utilize Different Carbohydrate Sources. Journal of Nutrition, 119(5), 727-733. https://10.1093/jn/119.5.727

Kamalam, B.S., Medale, F., Panserat, S., 2017. Utilisation of dietary carbohydrates in farmed fishes: new insights on influencing factors, biological limitations and future strategies. Aquaculture. 467, 3-27. https://10.1016/j.aquaculture.2016.02.007

Lee, S.M., Lee, J.H., 2004. Effect of dietary glucose, dextrin and starch on growth and body composition of juvenile starry flounder Platichthys stellatus. Fisheries Science, 70(1): 53-58. https://10.1111/j.1444-2906.2003.00770.x 
Leggatt, R.A., Raven, P.A., Mommsen, T.P., Sakhrania, D., Higgsa, D., Devlina, R.H., 2009. Growth hormone transgenesis influences carbohydrate, lipid and protein metabolism capacity for energy production in coho salmon (Oncorhynchus kisutch). Comparative biochemistry and physiology. Part B, Biochemistry \& molecular biology 154(1): 121-133. https://10.1016/j.cbpb.2009.05.010

Li, X.S., Zhu, X.M, Han, D, Yang, Y.X., Jin, J.Y., Xie, S.Q., 2014. Carbohydrate utilization by herbivorous and omnivorous freshwater fish species: a comparative study on gibel carp (Carassius auratus gibelio. var CAS III) and grass carp (Ctenopharyngodon idellus). Aquaculture Research, 47: 128-139. https://doi.org/10.1111/are.12476

Li, J.N., Xu, Q.Y., Wang, C.A., Wang, L.S., Zhao, Z.G., 2015. Effects of dietary glucose and starch levels on the growth, haematological indices and hepatic hexokinase and glucokinase mRNA expression of juvenile mirror carp (Cyprinus carpio). Aquaculture Nutrition, 22(3): 550-558. https://doi.org/10.1111/anu.12278

Li,S.L., Li Z.Q., Zhang, J.C., Sang, C.Y., Chen,N.S., 2019.The impacts of dietary carbohydrate levels on growth performance, feed utilization, glycogen accumulation and hepatic glucose metabolism in hybrid grouper (Epinephelus fuscoguttatus $\$ \times E$. lanceolatus $\left.\sigma^{7}\right)[\mathrm{J}]$. Aquaculture, 512:734351-. https://doi.org/10.1016/j.aquaculture.2019.734351

Li, X.Y., Zheng, S.X., Ma, X.K., Cheng, K.M., Wu, G.Y., 2020. Effects of dietary starch and lipid levels on the protein retention and growth of largemouth bass (Micropterus salmoides ). Amino Acids, 52(6): 999-1016. https://10.1007/s00726-020-02869-6

Luo, Y., Wu, X.Y., Li, W.F., Jiang, S.T., Lu, S.D., Wu, M.J., 2016. Effects of Different Corn Starch Levels on Growth, Protein Input, and Feed Utilization of Juvenile Hybrid Grouper (male Epinephelus lanceolatus $\times$ female $E$. fuscoguttatus)

North American Journal of Aquaculture, 78(2): 68-173. https://doi.org/10.1080/15222055.2015.1129004

Ma, H.J., Mu, M.M., Pu, D.C., Lin, S.M., Chen,Y.J., Luo, L., 2019. Effect of dietary starch level on growth, metabolism enzyme and oxidative status of juvenile largemouth bass, Micropterus salmoides. Aquaculture, 498: 482-487. https://doi.org/10.1016/j.aquaculture.2018.07.039

Mohanta, K.N., Mohanty, S.N., Jena, J.K., 2007. Protein-sparing effect of carbohydrate in silver barb, Puntius gonionotus fry. Aquaculture Nutrition, 13(4): 311-317. https://doi.org/10.1111/j.1365-2095.2007.00482.x

Mohanta, K.N., Mohanty, S.N., Jena, J.K., Sahu, N.P., Patro, B., 2009. Carbohydrate level in the diet of silver barb, Puntius gonionotus (Bleeker) fingerlings: effect on growth, nutrient utilization and whole body composition. Aquaculture Research, 40(8): 927-937. https://doi.org/10.1111/j.1365-2109.2009.02186.x

Nam, Y.K., Noh, J.K., Cho, Y.S., Cho, H.J., Cho, K.N., Kim, C.G., 2001. Dramatically accelerated growth and extraordinary gigantism of transgenic mud loach Misgurnus mizolepis. Transgenic Research, 10(4): 353-362. https://10.1023/A:1016696104185

Panserat, S., Kamalam, B.S., Fournier, J., Plagnes-Juan, E., Woodward, K., Devlin, R.H., 2014. Glucose metabolic gene expression in growth hormone transgenic coho salmon. Comparative Biochemistry and Physiology A-molecular \& Integrative Physiology, 170: 8-45. https://10.1016/j.cbpa.2014.01.013

Polakof, S., Panserat, S., Soengas, J.L., Moon, T.W., 2012. Glucose metabolism in fish: a review. J. Comp. Physiol. B. 182, 1015-1045. https:10.1007/s00360-012-0658-7

Rahman, M.A., Ronyai, A., Engidaw, B.Z., Jauncey, K., Hwang, G.L., Smith, A., 2001. Growth and nutritional trials on transgenic Nile tilapia containing an exogenous fish growth hormone gene. Journal of Fish Biology, 59(1): 62-78. https://10.1111/j.1095-8649.2001.tb02338.x

Saltiel, A.R., Kahn, C.R., 2001. Insulin signalling and the regulation of glucose and lipid metabolism. Nature 414(6865): 99-806. https://10.1038/414799a

Su, J.Z., Mei, L.Y., Xi, L.W., Gong,Y.L., 2020. Responses of glycolysis, glycogen accumulation and glucose-induced lipogenesis in grass carp and Chinese longsnout catfish fed high-carbohydrate diet. Aquaculture, 533. https://doi.org/10.1016/j.aquaculture.2020.736146

Song, X.R., Han, D., Liu, H.K., Yang, Y.X., Jin, J.Y., Zhu, X.M., Xie,S.Q., 2019. Regulations on glucose metabolism affected by dietary carbohydrate in different strains of juvenile gibel carp (Carassius gibelio). Aquaculture Research, 50(4): 1075-1086. https:// 10.1111/are.13981

Suárez, M.D., Sanz. A., Bazoco, J., García-Gallego, M., 2002. Metabolic effects of changes in the dietary protein: carbohydrate ratio in eel (Angilla anguilla) and trout (Oncorhynchus mykiss). Aquaculture International, 10(2): 143-156. https://10.1023/A:1021371104839

Wilson, R.P., 1994. Utilization of dietary carbohydrate by fish. Aquaculture 124(1-4): 67-80. https://doi.org/10.1016/0044-8486(94)90363-8 
Xia, B., Gao, Q.F., Wang, J., Li, P., 2015. Effects of dietary carbohydrate level on growth, biochemical composition and glucose metabolism of juvenile sea cucumber Apostichopus japonicus (Selenka). Aquaculture, 448: 63-70. https://10.1016/j.aquaculture.2015.05.038

Zhang, L.L., Zhou, Q., Cheng, Y., 2009. Effect of dietary carbohydrate level on growth performance of juvenile spotted Babylon (Babylonia areolata Link 1807). Aquaculture, 295(3): 238242. https://10.1016/j.aquaculture.2009.06.045

Zhang, Y., Liu, Y., Li, X., Gao, W., Wang, X., 2013. Effects of insulin and IGF-I on growth hormone- induced STAT5 activation in 3T3-F442A adipocytes. Lipids in Health and Disease, 12(1): 56-56. https://10.1186/1476-511X-12-56

Zhong, C.R., Song, Y.L., Wang, Y.P., Li, Y.M., Liao, L.J., Xie, S.Q., Zhu, Z.Y., Hu, W., 2012. Growth hormone transgene effects on growth performance are inconsistent among offspring derived from different homozygous transgenic common carp (Cyprinus carpio L.). Aquaculture, 356: 404411. https://doi.org/10.1016/j.aquaculture.2012.04.019

Zhou, C.P., Ge, X.P., Niu, J., Lin, H.Z., Huang, Z., Tan, X.H., 2015. Effect of dietary carbohydrate levels on growth performance, body composition, intestinal and hepatic enzyme activities, and growth hormone gene expression of juvenile golden pompano, Trachinotus ovatus. Aquaculture, 437: 390-397. https://doi.org/10.1016/j.aquaculture.2014.12.016 